\title{
Retracted: Braking Control System of Oilfield Minor Repair Machine Based on Wireless Sensor Network
}

\author{
Security and Communication Networks \\ Correspondence should be addressed to Security and Communication Networks; scn@hindawi.com
}

Received 7 April 2022; Accepted 7 April 2022; Published 17 May 2022

Copyright ( 2022 Security and Communication Networks. This is an open access article distributed under the Creative Commons Attribution License, which permits unrestricted use, distribution, and reproduction in any medium, provided the original work is properly cited.

Security and Communication Networks has retracted the article titled "Braking Control System of Oilfield Minor Repair Machine Based on Wireless Sensor Network" [1], due to concerns with the authenticity of the data. It was found that previous versions of this submission contained a figure unrelated to the topic of the paper and this graph was subsequently identified within several other submissions, all with accompanying text claiming to have generated the graph. A number of these submissions were rejected from the journal; however, 6 were published and have now been retracted from Security and Communication Networks and Scientific Programming [2-6].

The authors responded to explain that an author from one of the identified submissions had provided copy editing for their manuscript and introduced the graph and accompanying text in error.

The authors were unable to provide copies of correspondence to support their claim or the raw data from their study. The authors' explanation did not satisfy the concerns of the editorial board, and the article is therefore being retracted due to concerns with the reliability of the data.

The authors do not agree to the retraction.

\section{References}

[1] L. Zhuang, Y. Qi, and F. Zhang, "Braking Control System of Oilfield Minor Repair Machine Based on Wireless Sensor Network," Security and Communication Networks, vol. 2021, Article ID 6966041, 14 pages, 2021.

[2] L. Keshun, Z. Xijun, and Z. Xing, "Research on Analysis and Classification of Vulnerability of Electromagnetic Pulse with a STM32 Single-Chip Microcomputer," Scientific Programming, vol. 2021, Article ID 6836158, 10 pages, 2021.
[3] L. Huo, "Intelligent Recognition Method of Vehicle Path with Time Window Based on Genetic Algorithm," Security and Communication Networks, vol. 2021, Article ID 3614291, 11 pages, 2021.

[4] L. Fan, M. Xia, P. Huang, and J. Hu, "Research on Educational Information Platform Based on Cloud Computing," Security and Communication Networks, vol. 2021, Article ID 3109473, 11 pages, 2021.

[5] L. Yan, "Research on Crude Oil Trade Procurement Model Based on DEA-Malmquist Algorithm," Scientific Programming, vol. 2021, Article ID 6360439, 10 pages, 2021.

[6] G. Yin, R. Qi, J. Zhao, X. Zhao, and J. Xu, "Optimization Method of Sports Service Network Node Layout Based on Network Communication," Security and Communication Networks, vol. 2021, Article ID 3013135, 8 pages, 2021. 


\title{
Braking Control System of Oilfield Minor Repair Machine Based on Wireless Sensor Network
}

\author{
Li Zhuang $(\mathbb{D}$, Yaoguang Qi, and Fenna Zhang \\ College of Mechanical \& Electrical Engineering, China Univiersity of Petroleum (Eastern China), Dongying, China
}

Correspondence should be addressed to Li Zhuang; b14040123@s.upc.edu.cn

Received 25 July 2021; Revised 20 August 2021; Accepted 25 August 2021; Published 8 September 2021

Academic Editor: Jian Su

Copyright (c) $2021 \mathrm{Li}$ Zhuang et al. This is an open access article distributed under the Creative Commons Attribution License, which permits unrestricted use, distribution, and reproduction in any medium, provided the original work is properly cited.

\begin{abstract}
Traditional minor repair machines have low control efficiency and low automation. In order to improve the brake control efficiency of the oilfield minor repair machine, the oilfield minor repair machine brake control system based on wireless sensor network is designed. The front-end controller collects the data of the oilfield repair machine through the wireless sensor and realizes the communication between the sensor nodes and the data interaction with the communication node through the field bus inside the equipment. The server uploads the processed data through the $3 \mathrm{G}$ network, and the server receives the data from the front-end controller and saves it in the database. The remote-control center accesses the server through the Internet to view the front-end controller data. The brake control module uses the expert fuzzy PID control method to control the speed, oil pressure, and operating status of the oilfield minor repair machine and sends the data to the front-end control center to complete the oilfield minor repair remote-control machine by sending control instructions. Experimental results show that the system can meet the braking control requirements of oilfield minor repair machines, the output can quickly track input changes, the braking control effect is good, the dynamic deviation of the machine can be reduced, and the braking control efficiency can be improved.
\end{abstract}

\section{Introduction}

With the rapid development of China's economy, the oilfield minor repair machine plays an increasingly important role in the oilfield minor repair operation. All kinds of oilfield minor repair machines are becoming more and more of high-speed, large-scale, complex, and heavy-duty, and the automation level of the unit has been greatly improved [1-3]. The equipment cost, maintenance cost, and management cost of oilfield minor repair machine have been greatly increased. To solve the above problems, we have to introduce the current advanced information and intelligent new technology into the traditional maintenance and management of oilfield minor repair machine. In today's society, the scope of use of oilfield minor repair machine is more extensive, and the frequency of use is more frequent, which puts forward higher requirements for the reliability and stability of the equipment, and the corresponding equipment condition monitoring and braking control become more complex. First of all, the oilfield repair machine is developing towards electrification. The corresponding equipment monitoring should not only monitor the mechanical condition of the equipment, but also monitor the electrical signal. It needs a wide range of knowledge, and the on-site technicians do not necessarily have the required professional knowledge. However, the professionals with the technology are generally concentrated in Colleges and research institutes, and the unreasonable distribution of human resources also hindered the promotion and application of monitoring and diagnosis technology of oilfield minor repair machine [4]. If the oilfield minor repair machine has a serious failure, it needs the help of relevant experts to solve the problem, but if every time there is a failure, professionals will be invited to the scene, which is not very realistic, but also a waste of social resources. Therefore, the condition monitoring and braking control of oilfield minor repair machine equipment put forward new technical requirements, and the rapid development of information technology and digital technology provides solutions for the new problems faced by oilfield minor repair machine. The 
improvement of information technology in today's society has broken the limitation of traditional communication mode [5], making information exchange more free, fast, and convenient. The remote condition monitoring and fault diagnosis equipment relying on information technology can transmit the field operation data of oilfield minor repair machine to the server in real time. The equipment data stored in the server is analyzed and processed based on big data technology, and the data will be transmitted to the server when the processing information is sent to the remote control. The remote-control terminal will warn the equipment user and give the processing scheme according to the processing data and lock and alarm the equipment with security problems. It is found that the topology of data communication network roles is an important factor in its security robustness against attack. In such networks, by changing the topology, the security robustness against intentional attack that aims at bringing down network nodes may vary. This bringing down is a kind of destruction and interruption threat that attacks the availability of the network (i.e., attack on network resources and links).

Oilfield minor repair operation is a set of technology that is produced with the development and production of the oilfield, maintains the normal work of oil and water wells in the oilfield, and provides efficient operation guarantee for oil and water wells in the oilfield [6]. With the continuous update and progress of oilfield production technology, workover technology must also make corresponding progress. In recent years, with the continuous development of computer technology and network technology, information management system has been a good development. In order to improve the working efficiency and level of oilfield, oilfield production is becoming more and more information-based. With the informatization of oilfield production, the management of oilfield minor workover operations has been completely operated by hand at the beginning, and now some workover tools have been controlled by computer instead of manual operation. With the continuous advancement of information technology, a large amount of information data must be processed in oilfield production. Oilfield minor repair operation also needs to control a lot of data information, and the traditional manual control is not suitable for the new requirements.

However, in the past, the braking control system of oilfield minor repair machine is extremely limited. For example, Tang Nana et al. used the 4G-LTE control system [7]. In the braking control process of oilfield minor repair machine, the calculation of the system is complicated, which leads to the poor braking control efficiency of oilfield minor repair machine. Xiao et al. designed the control system based on motion controller. In the process of dynamic control of the oilfield minor repair operation, due to the low degree of intelligence of the system, the braking control effect of oilfield minor repair machine was not ideal [8]. Wireless sensor network refers to the combination of various peripheral information acquisition devices, such as infrared sensors, temperature sensors, speed sensors, laser scanners, and other devices with the Internet to form a large network. Sensors are embedded in objects that need to be detected, such as roads, bridges, railways, and water supply systems. After forming a wireless sensor network through Internet communication, a large number of sensors are embedded in the network. After the formation of wireless sensor network through Internet communication, a large amount of data is processed through cloud storage technology, and big data analysis is carried out through cloud computing technology, so as to integrate wireless sensor network and realize intelligent control and management. Therefore, wireless sensor network can measure the sonar, thermal, infrared, and radar signals in the surrounding environment or the measured objects with the help of sensors in the sensing layer, so as to detect the material phenomena including temperature, humidity, noise, light intensity, pressure, size, speed, and direction of moving objects. Through the collection of these data, it is possible for the network to control all kinds of environment and equipment in real time, which greatly expands the application scope of network technology. Wireless sensor network technology is rated as the first of the ten technologies that will change the world in the future by technology review of the United States and points out that the information age will change from "Network is computer age" to "Network is sensor age." According to the concept of wireless sensor network provided by open Geospatial Information Alliance, sensors are widely used in traffic management, health care, manufacturing, construction, and other aspects, so wireless sensor networks can also be called "Internet of things." At present, some foreign researches have introduced wireless sensor network technology into petroleum engineering related fields. For example, Khoshraftar et al. proposed wireless sensor networks to replace the existing manually controlled oil pump system [9]; Liu et al. proposed to use Sprouts WSN platform to complete three kinds of parameter monitoring of shovel teeth in oil sand production business [10]; Cui et al. proposed to infiltrate ZigBee technology into engineering control field [11]; Ying proposed a wireless monitoring scheme for oil and gas pipeline damage and leakage [12]. Generally speaking, although these studies involve wireless sensor networks in logging field, they mainly focus on the original engineering signal acquisition and rarely focus on the braking control of oilfield minor repair machine.

To sum up, the contribution of our method can be described as follows: the braking control system of oilfield minor repair machine based on wireless sensor network is designed. The system integrates mobile communication, remote positioning, data processing, and other technologies. Relying on the basic framework and core technology of wireless sensor network, the system realizes the acquisition, analysis, and remote control of the status information of the oilfield minor repair machine and uploads and analyzes the location, equipment status, fault information, and other data of the oilfield minor repair machine in real time. The data information of maintenance operation is stored in the database of the control system. The management personnel can analyze the data information in time, brake and control the oilfield minor repair machine, ensure the safe work of the oilfield minor repair machine, and improve the braking 
control efficiency and management level of the oilfield minor repair machine.

This paper is divided into 4 sections. Section 2 gives a brief introduction about VRPSTW. Our proposal and the experimental results can be described in Section 3 in detail. Section 4 concludes this paper with contributions, limitations, and future works. The research contributions of the thesis include the following points:

(1) Propose the design of brake control based on wireless sensor network

(2) Analyze the front-end controller data to realize the communication between sensor nodes

(3) The brake control module is used to control the speed, oil pressure, and the operating status of the oilfield minor repair machine

\section{Braking Control System of Oilfield Minor Repair Machine Based on Wireless Sensor Network}

2.1. Overall Structure of the System. According to the needs of oilfield minor repair operation, terminal equipment needs to be installed on the oilfield minor repair machine. Through the terminal equipment and the server, the equipment identity is bound to provide each oilfield minor repair machine with a unique identity for users to query. The oilfield minor repair machine for outdoor operation needs to track and analyze the location information of the equipment and transmit the location information to the server in real time. At the same time, the control system needs to collect the status information of the oilfield minor repair machine through wireless sensors and front-end instruments, and the terminal equipment needs to upload and analyze the equipment status information and fault information of the oilfield minor repair machine in real time. In addition, the braking control system needs to control the oilfield minor repair machine through the remote-control terminal to improve the management efficiency of the operator. According to the above analysis, a braking control system of oilfield minor repair machine based on wireless sensor network is designed. The system is required to have the characteristics of portability, high integration, rapid response, and tailoring configuration. It mainly includes sensor network, front-end controller, remote-control center, and field control terminal. This paper focuses on the development and research of nuclear technology such as acquisition, receiving, analysis, processing, and transmission of monitoring signal in control system.

In this paper, we utilize the entropy loss function to build the model for our research problems. It can be defined as follows: loss $(x, y)=\sum_{i=1}^{n}-p_{i} \log \left(1-p_{i}\right)$, where $x$ and $y$ are represented as the real arts and crafts' score and difficulty, and $y$ means the predicted score and difficult of our proposal. $P_{i}$ means their probability when they are similar. The bigger the value of the loss, the worse our proposal performed. And our proposal is used to train a model that fits the real integrated oilfield and repaired oilfield, so that the machine can assist the designed oilfields. The overall structure of the braking control system of the oilfield minor repair machine based on wireless sensor network is shown in Figure 1.

It can be seen from Figure 1 that the sensor network composed of wireless sensor nodes is used as the physical layer to complete the data acquisition of oilfield minor repair machine, and the communication between sensor nodes and the data interaction with communication node (front-end controller) is realized through the field bus inside the equipment. As a communication node, the front-end controller also has the function of collecting and processing positioning information and part of the equipment status monitoring information and then uses $3 \mathrm{G}$ network to upload data. At the same time, the front-end controller communicates with the sensor network to complete the equipment control task. The server receives the data from the front-end controller and saves it in the database for big data analysis in the future. The remote-control center accesses the server through the Internet to view the data of the front-end controller and uses the braking control module to send control instructions [13] to the front-end controller to complete the remote control of the oilfield minor repair machine. At the same time, the field operation control terminal uses the local area network to view the video information of the oilfield minor repair machine in the frontend controller. In the last three years, many methods are proposed to handle the problem; here, we introduced three outstanding methods, that is, GNB, RNB, and CNB, which can be used to solve the related works taking different kinds of network structures. GNB is a graph-based network that builds connections between different risk nodes. RNB uses a specific loss structure to keep the similarity of real and predicted crafts design. CNB is the basic model that needs more computation consumption to obtain the desired performances. However, these methods have these disadvantages, respectively. GNB is too slow, RNB is so complicated, and CNB also needs more spaces. Compared with these methods, our proposal can work effectively well for oilfield identification by using the evolving algorithm, which does not utilize the structure of network and is not time consuming at all. And our proposal can deal with the problems easily, and we also need a smaller computation space to build our model. However, our model may obtain a relatively lower accuracy than others, which may make the prediction unstable.

\subsection{Design of Wireless Sensor Network}

2.2.1. Structure of Wireless Sensor Network. Wireless sensor network technology is a new technology combining sensor technology, network communication technology, and microelectronics technology. The structure of wireless sensor network is shown in Figure 2.

As can be seen from the above figure, wireless sensor network is composed of sensor nodes and sink nodes. The sensor node is responsible for uploading the collected physical quantities in the form of data. The sink node is 


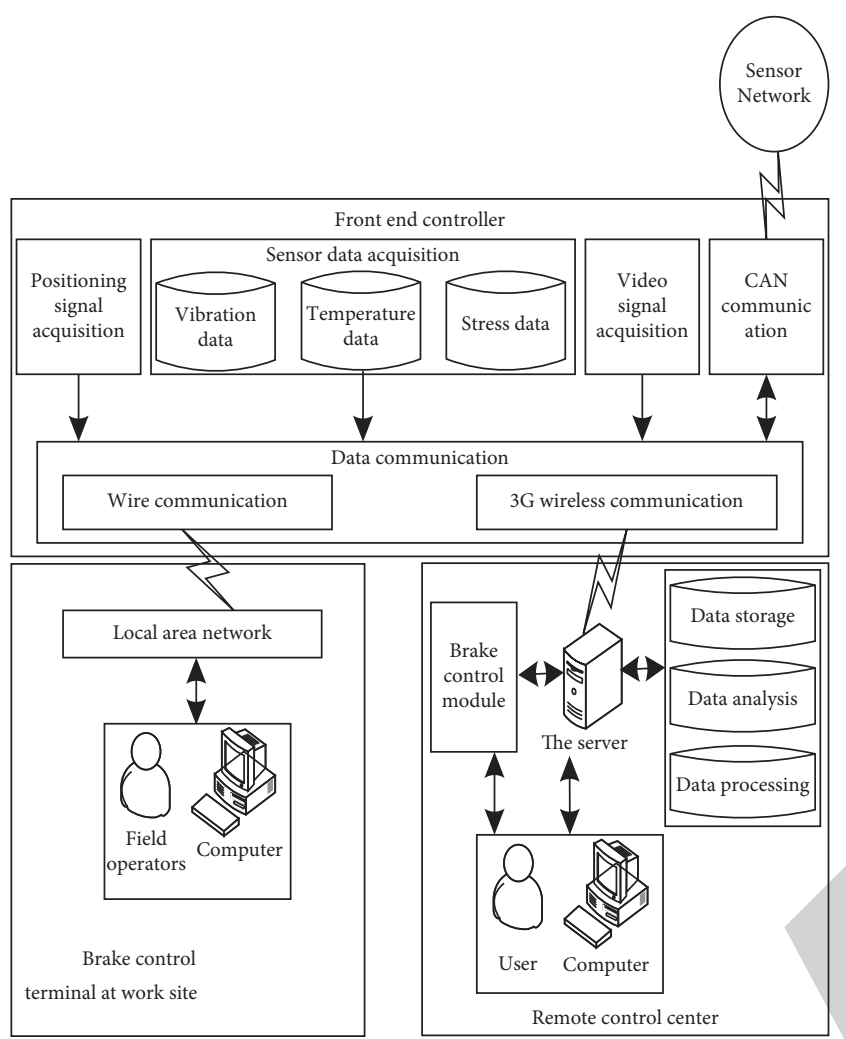

FIGURE 1: Overall structure of the system.

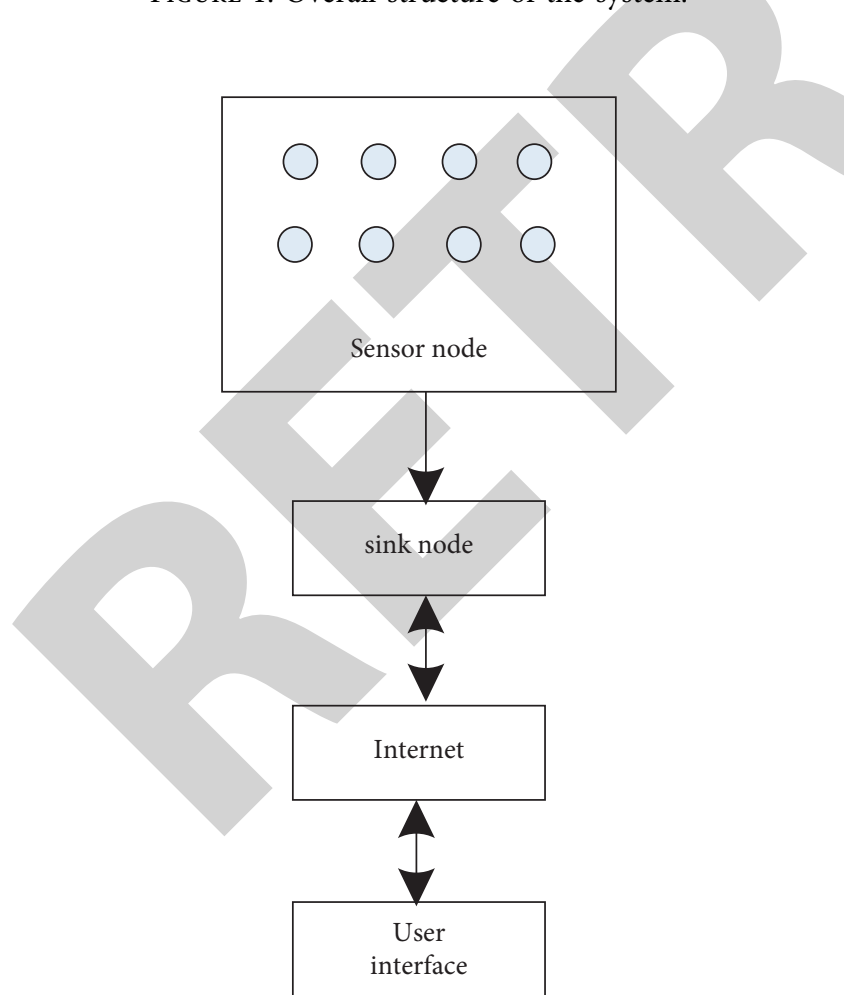

FIgURE 2: Structure of wireless sensor network.

responsible for managing the sensor nodes in the area and making them form a network in the form of self-organization. The information collected by the sensor nodes is uploaded to users through the Internet.
2.2.2. Topology of Wireless Sensor Networks. The foundation of ZigBee communication protocol is IEEE802.15.4, which is a protocol standard of IEEE wireless personal area network working group. ZigBee wireless sensor network is used to build wireless network for each oilfield minor repair unit to realize the networking of wireless sensor network. In ZigBee wireless sensor network, three types of devices are defined according to different network roles: End Device, Router, and Coordinator; according to different communication capabilities, they are divided into two different functional devices: full-function device (FFD) and reduced function device (RFD). FFD devices become full-function devices, because they can communicate not only with FFD devices, but also with RFD devices. However, RFD devices cannot communicate with each other directly; they must be forwarded by FFD devices. A coordinator is the total controller of a network, and it must be FFD. In all ZigBee wireless sensor networks, there must be a coordinator, and there can only be one coordinator. The role of coordinator in the network includes the following: selecting the channel used by the network, starting the network, allowing the subnodes to connect to the network, delivering messages, and security management. A router must exist in tree network and mesh network, and it must also be FFD. The role of router in network includes the following: routing messages, allowing child nodes to connect to the network. The terminal device can be FFD or RFD, which is used as a specific application device in the network [14]. A terminal device is usually battery-powered, so the power consumption design needs special consideration. When there is no message sent or received, it can enter the sleep state to save power. Terminal devices cannot forward messages, nor can they allow child nodes to access the network through them.

In the networking mode, ZigBee wireless sensor network supports tree network topology and mesh network topology. The schematic diagram of each structure is shown in Figure 3.

As can be seen from the figure above, the tree topology consists of a coordinator and a series of terminal device nodes and routing nodes. In tree topology, the coordinator and routing node can contain their own child nodes, but terminal devices cannot have their own nodes. In tree topology, each node can only communicate with its parent node and child node. If data is sent from one terminal device node to another terminal device node, it will be transmitted up the tree path to the nearest first node, and then down to the target node [15]. The mesh topology consists of a coordinator and a series of terminal device nodes and routing nodes. This network topology is similar to the tree topology, but the mesh topology has more flexible routing rules, and the routing nodes can communicate directly. This structure can make the routing mechanism more efficient and more robust. If there is a problem with one route, the message can be automatically transmitted along the other routes. In the network supporting mesh topology, the network layer will provide the corresponding routing exploration function, which can make the network layer find the optimal path. 


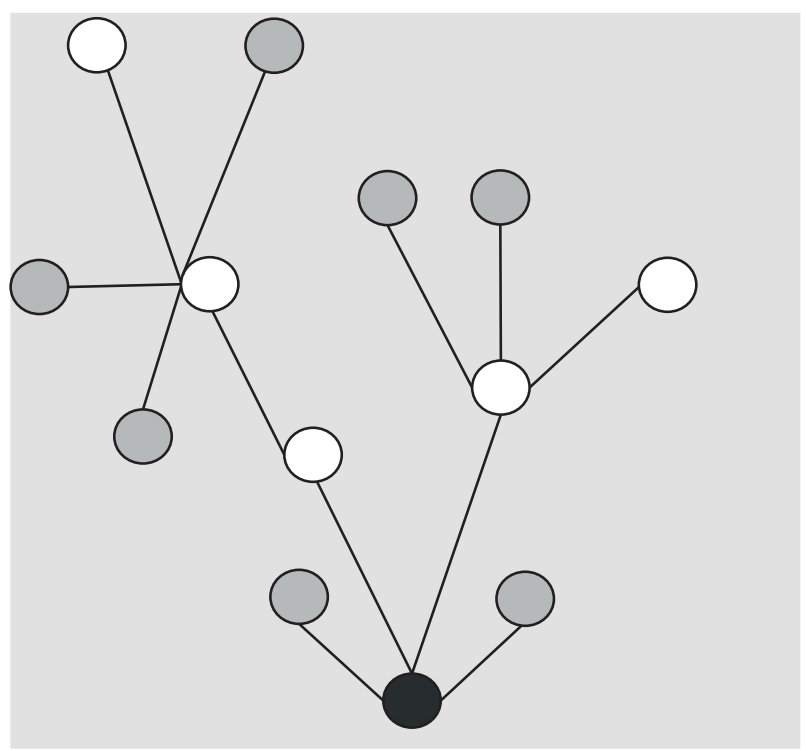

(a)

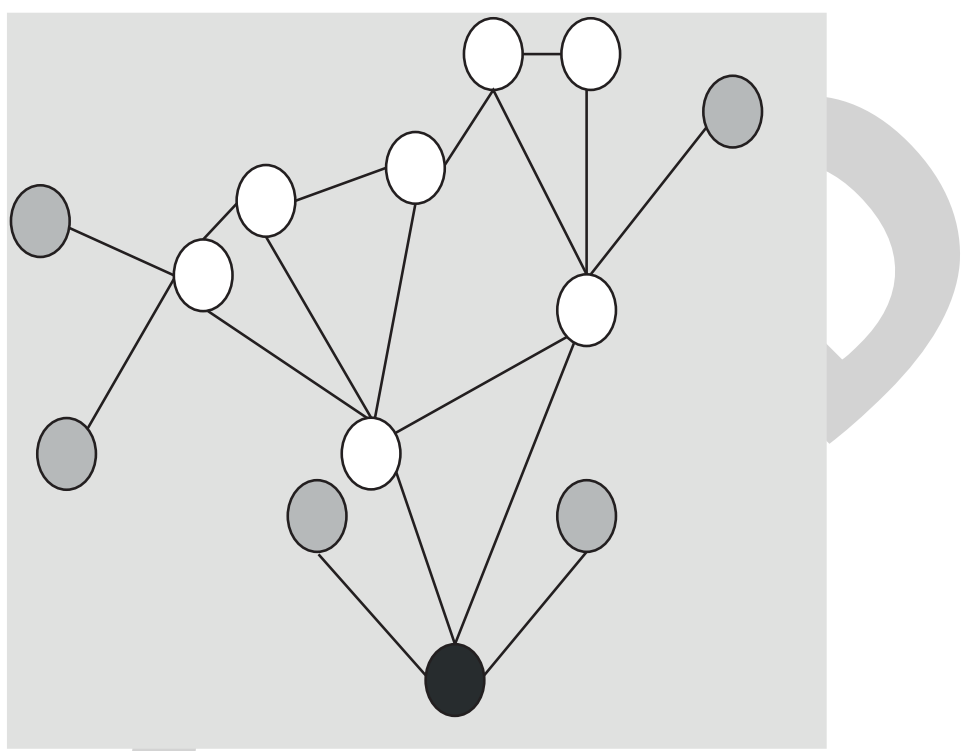

(b)

Figure 3: Zig Bee topology of wireless sensor network. (a) Tree network topology. (b) Mesh networking topology.

\subsection{Design of Communication Module Program}

2.3.1. Design of CAN Communication Program. The CAN bus in the system can be used as a wired communication mode of information exchange between sensor nodes and between sensor nodes and communication modules. The sensor can realize the data connection between sensor and control center with the help of CAN bus. The oilfield minor repair machine needs to realize the locking function and also needs to realize the control operation through CAN bus. The flowchart of CAN communication is shown in Figure 4. After the program starts, the CAN interface is initialized. The device node of CAN interface of front-end controller is CANO, so CAN0 is set here.

As can be seen from the above figure, after the CAN interface is initialized with the IP instruction of Linux, the program needs to query whether the device is bound on the server, and the device can only execute the CAN communication program after binding the server. Creating a Linux system in front-end controller config.txt. as a lock function status file of storage device, when the lock function status changes, it will modify the engine status flag bit in the config.txt. file. Therefore, it should firstly query the engine status flag in config.txt. file. According to the bit information in the file, CAN bus sends the locking function status information to the engine. After that, the program judges whether there is a command to change the status information of vehicle locking function (speed limit/shutdown). If there is, it will change the flag bit in the config.txt. file and then execute the instruction. If there is no command to change the status information of vehicle locking function (speed limit/shutdown), the sensor network data will be read from the bus. Finally, it can judge whether there is a command to turn off CAN communication. If not, continue to run CAN communication program to ensure that the front-end controller continuously sends CAN packets to the engine [16].
2.3.2. Design of Wireless Communication Module. The wireless communication module is the bridge between the front-end controller and the server. The front-end controller transmits the positioning, working status, and other information of the oilfield minor repair machine to the server through the wireless communication module. The front-end controller can also receive the control information from the control center through the wireless communication module to realize the braking control operation of the oilfield minor repair machine, such as shutdown and speed limit. The core of wireless communication module is also $3 \mathrm{G}$ communication module SIM5320, which communicates with server through 3G network. 3G communication refers to the high-speed data transmission of cellular mobile communication technology, which perfectly realizes the seamless roaming of the world [17], and can process a variety of media information including music, images, and video streams and provide a variety of information services including teleconference, web browsing, and e-commerce. This design uses SIM5320 built-in TCP/IP protocol, and at the instruction set, it can support the front-end controller automatic networking, permanent online, offline reconnection, and other functions through the serial port and the main control chip for data interaction [18]. The workflow of wireless communication module is shown in Figure 5.

As can be seen from the above figure, after the wireless communication thread is started, the serial port of the main control chip is initialized first. Since the baud rate of SIM5320 is 115200 bps by default, and the serial port data format is 8-bit data bit, 1-bit start bit, and 1-bit end bit, this design will not be modified here, so the serial port is initialized according to this default value. The SIM5320 function is initialized and set to full-function Internet connection mode. After the thread of wireless communication module is created in the function, the function 


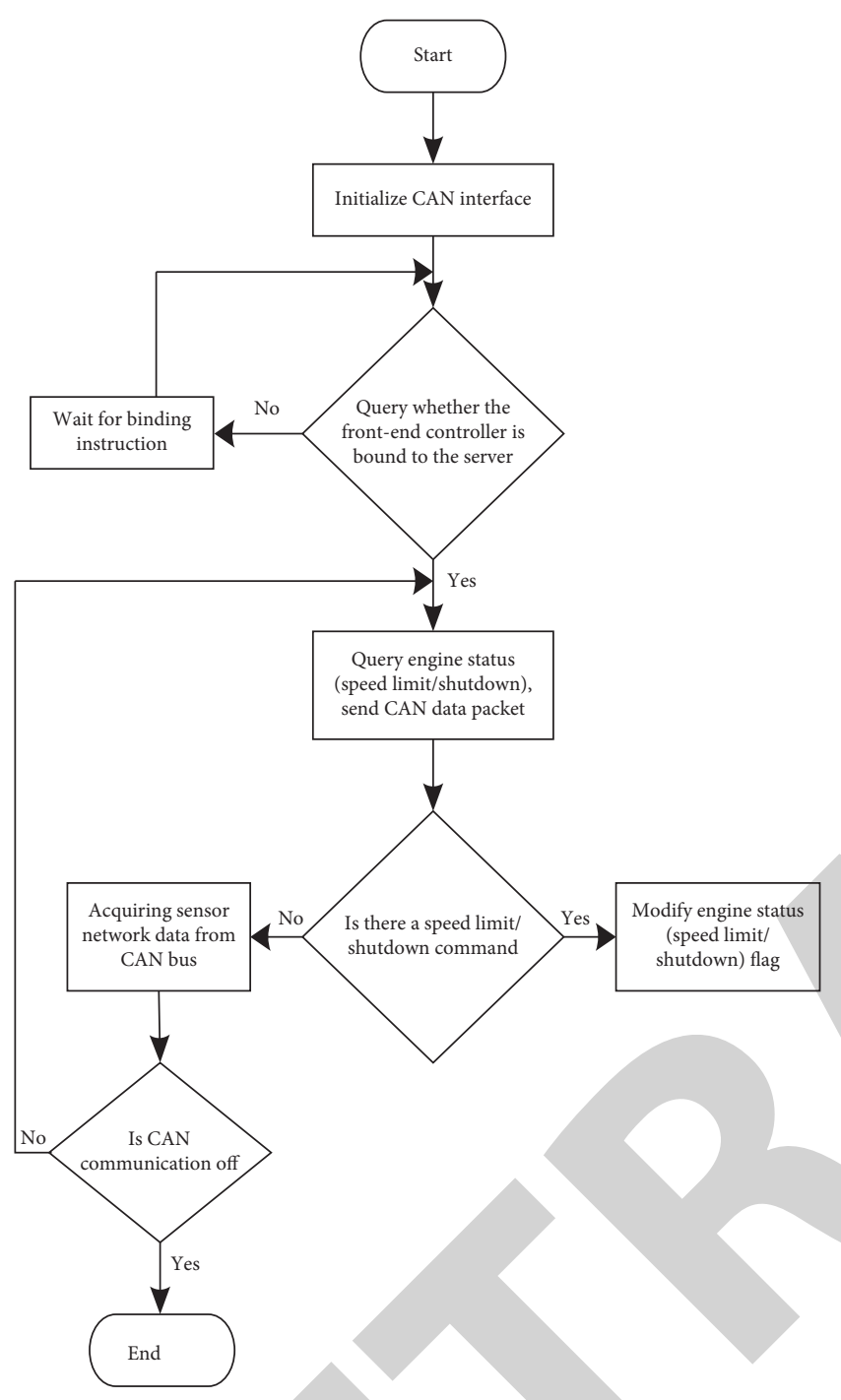

FIgURE 4: Flowchart of CAN communication.

wcdm_init() is used to register the $3 \mathrm{G}$ network. At the same time, the device hardware number IMEI, cellid, and location area code lac of the device SIM5320 can be obtained, to get the international mobile subscriber identity (IMSI) code from the SIM card and parse it into mc and mnc. After establishing a TCP connection with the server, it can judge whether the front-end controller is bound to the server. If it is not bound, it will not send and receive network packets. However, in order to ensure that the TCP connection with the server is continuously open, it will also skip packets every 5 seconds. If the device has been bound with the server, it will send and receive data. In order to realize the permanent online function of the device, the wireless communication device keeps sending and receiving data before the device receives the disconnection command from the server. When the device receives the disconnection command from the server, it disconnects the TCP connection and closes the wireless communication thread [19].

Are you spending money on energy unnecessarily? Did you know that, by implementing sensor technology, you will have a comprehensive understanding of where and how

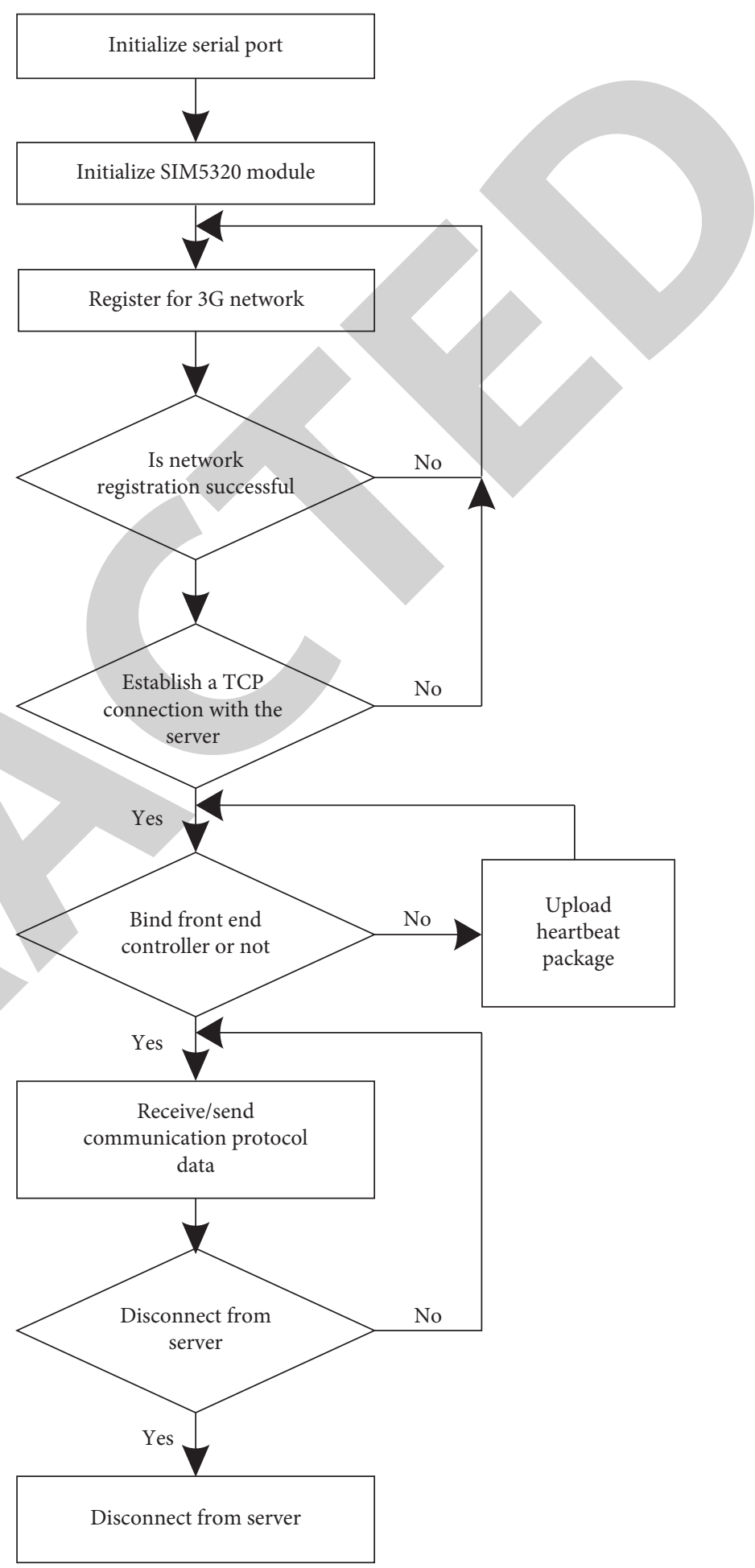

FIGURE 5: Workflow of wireless communication module.

much energy you are using? Combined with space occupancy data, you can use HVAC and lighting appropriately according to occupancy and needs.

By equipping your device with smart sensors, you can learn about its health status in real time. Sensors can be placed on the device to monitor thermal signals, establish baseline performance, and indicate when thresholds are 
exceeded. If a piece of equipment is operating abnormally, maintenance can be scheduled accordingly to prevent it from failing.

2.4. Braking Control Method. The braking control module adopts expert fuzzy PID control method to control the speed, oil pressure, and running state of the oilfield minor repair machine.

2.4.1. Principle of PID Control. PID control algorithm has the advantages of simple principle, being easy to use, and good robustness, which has been widely used in industrial process control [20]. If the improved PID control is included, about $90 \%$ of the control loops have PID structure. The conventional PID controller is a kind of linear controller, which is composed of proportional term $(P)$, integral term $(I)$, and differential term $(D)$. According to the given value $r(t)$ and the actual operation value $y(t)$ of the system, the analog PID controller forms the control deviation $e(t)$ and then controls the control object. The control law is shown in formulas (1) and (2):

$$
\begin{aligned}
& e(t)=r(t)-y(t), \\
& u(t)=K_{p}\left[e(t)+\frac{1}{T_{i}} \int_{0}^{t} e(t) \mathrm{d} t+T_{d} \frac{\mathrm{d} e(t)}{\mathrm{d} t}\right],
\end{aligned}
$$

in which the output of the controller is expressed in $u(t)$; the proportion coefficient is expressed by $K_{p}$; the integral time constant is expressed by $T_{i}$; the differential time constant is expressed by $T_{d}$.

With the development of electronic technology, PID control algorithm is realized by computer programming.
The computer uses the sampling period to discretize the control object and take sample s [21]. The analog PID control algorithm cannot be used directly, so it needs to be discretized to form a digital PID control algorithm, as shown in formulas (3) and (4).

$$
u(n)=K_{p}\left\{e(n)+\frac{T}{T_{i}} \sum_{j=0}^{n} e(j)+\frac{T_{d}}{T}[e(n)-e(n-1)]\right\},
$$

$$
u(n)=K_{p} e(n)+K_{i} \sum_{j=0}^{n} e(j)+K_{d}[e(n)-e(n-1)]
$$

in which the deviation value of the $n$-th sampling time of the computer is expressed by $e(n)$; the deviation value of the $n$-1-th sampling time of the computer is expressed by $e(n-1)$; the sampling period of the computer is expressed by $T$; the integral coefficient is expressed by $K_{i}$, and $K_{i}=K_{p} T / T_{i} ;$ the differential coefficient is expressed by $K_{d}$ and $K_{d}=K_{p} T_{d} / T$; the output value of the $\mathrm{n}$-th sampling time of the computer is expressed in $u(n)$.

Similarly, the control quantity of the $(n-1)$-th digital PID output can be obtained recursively, as shown in the following formula:

$$
u(n-1)=K_{p} e(n-1)+K_{i} \sum_{j=0}^{n-1} e(j)+K_{d}[e(n-1)-e(n-2)] .
$$

The incremental PID control algorithm can be obtained by subtracting formula (5) from formula (4), as shown in formulas (6) and (7).

$$
\begin{aligned}
\Delta u(n) & =K_{p}[e(n)-e(n-1)]+K_{i} e(n)+K_{d}[e(n)-2 e(n-1)+e(n-2)], \\
u(n) & =u(n-1)+\Delta u(n) .
\end{aligned}
$$

2.4.2. Expert Fuzzy PID Control. Combining the fuzzy control with the PID control, the expert fuzzy PID control method is designed. Fuzzy control is one of the important branches of intelligent control technology. It is a modern control theory based on fuzzy mathematics. Fuzzy control uses the theory and method of fuzzy mathematics to transform the state information of the controlled object into fuzzy language variable information $[22,23]$. The practical experience of field operators and the theoretical knowledge of relevant experts are summarized as fuzzy reasoning rules in the form of fuzzy language, which simulates the thinking form and thinking method of human beings to carry out fuzzy logic reasoning, so as to realize the control of the controlled object. The structure of expert fuzzy PID controller is shown in Figure 6.

The design of expert fuzzy PID control will be divided into five parts: controller structure design, input fuzzy interface design, fuzzy rule design, fuzzy reasoning and defuzzification design, and expert control rule design. Generally, the dimension of fuzzy controller is divided according to the number of input variables. Fuzzy control can be divided into one-dimensional, two-dimensional, three-dimensional, and multidimensional fuzzy controllers. The two-dimensional fuzzy controller selects the deviation $e$ and the deviation change rate $e c$ of the controlled variable and the given variable as the input of the controller. The deviation $e$ and the deviation change rate $e c$ are easy to obtain and describe the dynamic characteristics of the control process accurately and comprehensively to a large extent. The control effect is better than that of the one-dimensional fuzzy controller, and the computational complexity is far less than those of the three-dimensional and multidimensional fuzzy controllers. In order to simplify the calculation and ensure the accuracy of the system control, 


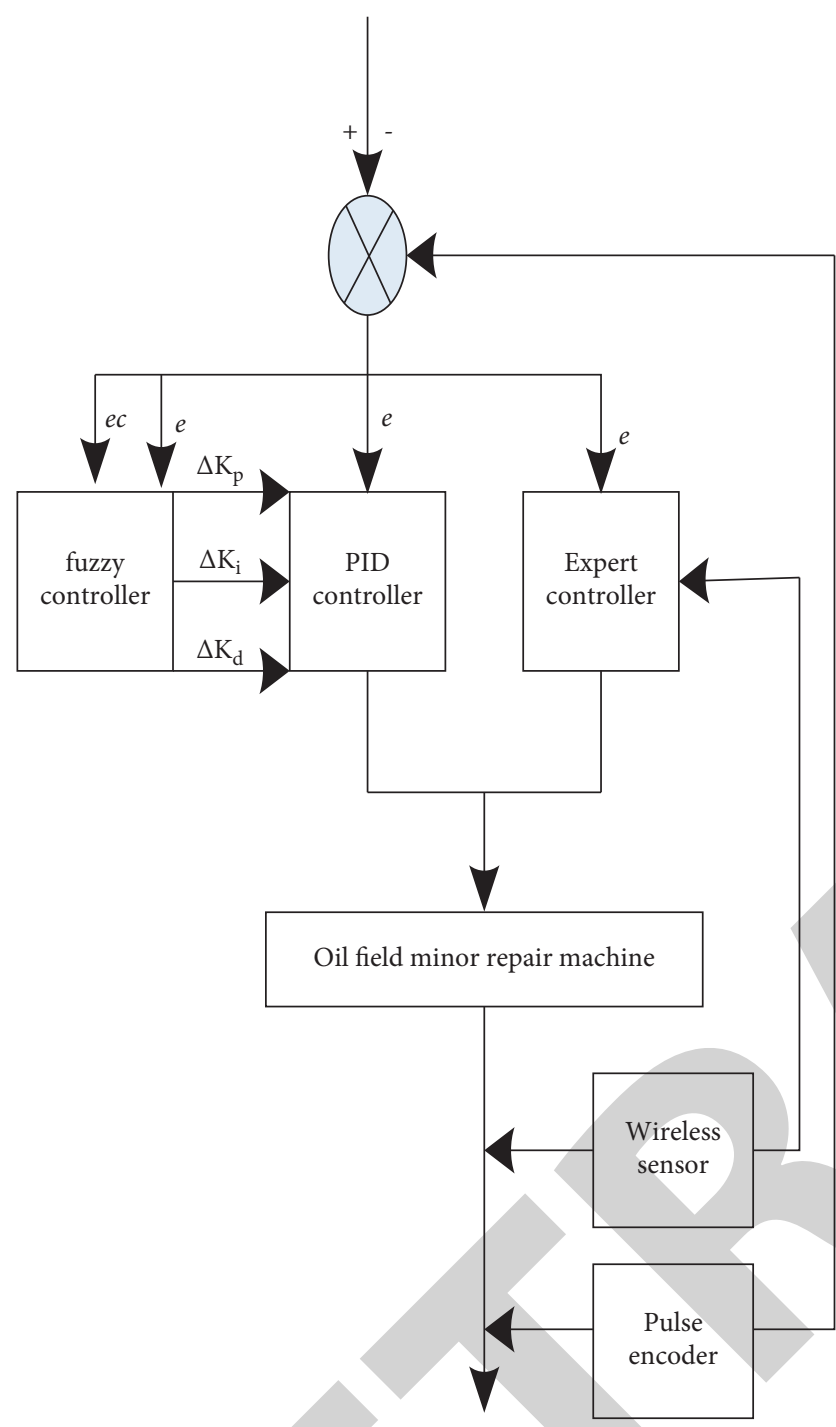

FIgURE 6: Structure of expert fuzzy PID controller.

the system in this paper uses two-dimensional fuzzy controller. Through the input of error $e$ and error change rate $e c$, combined with fuzzy rule reasoning based on expert experience, the PID controller parameters are adjusted online by modifying the proportional coefficient increment $\Delta K_{p}$, integral coefficient increment $\Delta K_{i}$ and differential coefficient increment $\Delta K_{d}$. In order to make the control process safer and more reliable, this paper uses incremental PID control algorithm to realize the braking control of oilfield minor repair machine.

In the last three years, many methods are proposed to handle the problem of braking control system of oilfield minor repair machine based on deep network. Here, we introduced three outstanding methods, that is, Graphcontrol [9], Reset_control [10], and CNN-control [11], which can be used to solve the related works taking different kinds of network structures. The first one is a graph-based network that builds connections between different risk nodes and connected each component according to their interrelationships. And the second one uses a specific loss structure to keep the similarity of real and predicted crafts design. The last one is the basic model that needs more computation consumption to obtain the desiring performances. However, these methods have these disadvantages, respectively. The Graph-control is too slow, the Resetcontrol is so complicated, and the CNN-control also needs more spaces. Compared with these methods, our proposal can work effectively well by using the wireless sensor equipment, which does not utilize the structure of network and is not time consuming at all. Importantly, it can give the feedback as soon as possible.

\section{Experimental Analysis}

In order to verify the effect of the optimized system in this paper, the Matlab platform was used to build an oilfield repair machine model to test the performance of the system. To investigate the effectiveness of our recommendations and other methods, here, we consider $F 1$-score to evaluate the experimental results, which can be defined as follows. We also compared the three methods proposed in the past three years (that is, GNB, RNB, and $\mathrm{CNB}$ ) with our recommendations to study the effectiveness of our method. All these methods are introduced in Section 3. In more detail, we use confusion matrix, accuracy, and precision to evaluate model performance. The matrix can be defined as follows:

$$
\begin{aligned}
\text { accuracy } & =\frac{\mathrm{TP}+\mathrm{TN}}{\mathrm{TP}+\mathrm{TN}+\mathrm{FP}+\mathrm{FN}}, \\
f 1-\text { score } & =\frac{2^{*} \text { precise }^{*} \text { recall }}{\text { precise }+ \text { recall }}, \\
\text { precise } & =\frac{\mathrm{TP}}{\mathrm{TP}+\mathrm{FP}}, \\
\text { recall } & =\frac{\mathrm{TP}}{\mathrm{TP}+\mathrm{FN}} .
\end{aligned}
$$

The response time comparison results of the three systems are described in Figure 7 (Table 1).

It can be seen from Figure 7 that the control response time of the system in reference and the system in reference does not change smoothly, and the transition time is long. Compared with the other two systems, the overshoot of the control system is also significantly reduced, and the dynamic and static characteristics of the control system are improved. In this paper, the system control can meet the braking control requirements, the output can quickly track the input changes, and the control effect is better than that of the other two systems.

In the strong jitter environment and weak jitter environment of transmission link environment of Rician channel and Gaussian channel, the data upload bandwidth of three systems is tested, and the test results are shown in Figure 8 .

As can be seen from Figure 8, the data upload bandwidth of the system in this paper in the two link jitter environments has significant advantages, the data upload bandwidth is higher, and the jitter situation is lower than the other two systems. This is because the system uses wireless sensor 

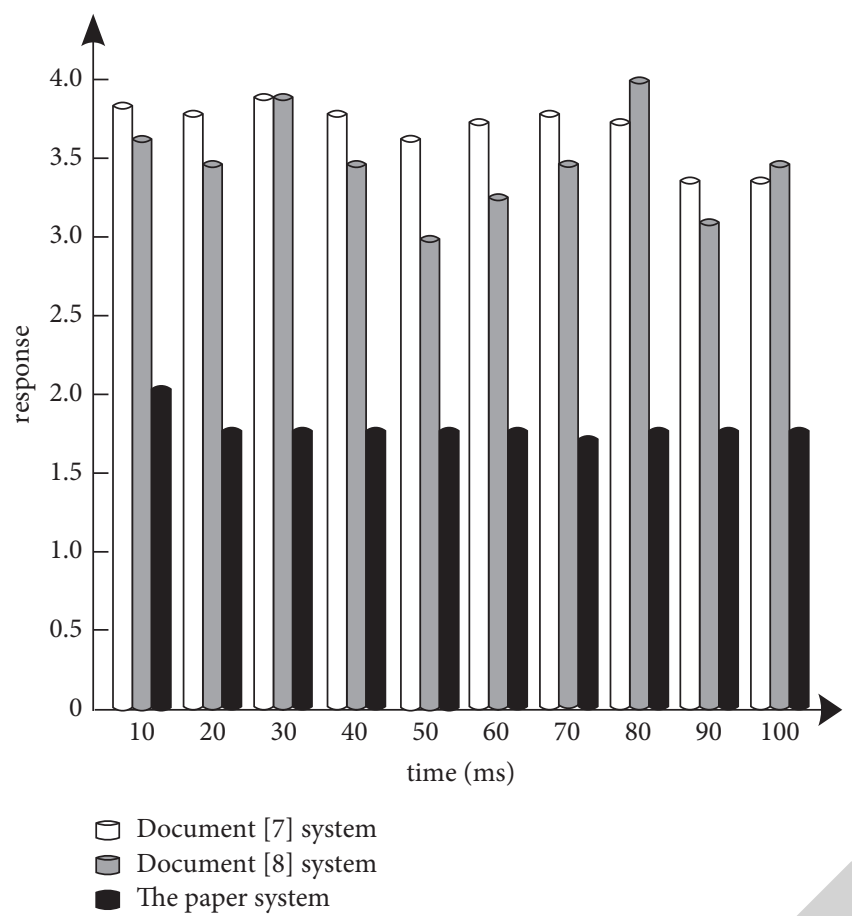

Figure 7: System response time.

TABLE 1: Actual forecast results.

\begin{tabular}{lcc}
\hline Real prediction & Positive & Negative \\
\hline Positive & TP & FN \\
Negative & FP & TN \\
\hline
\end{tabular}

networks, the communication module can significantly improve the data transmission at the same time, and the traditional solution is difficult to solve the problem of limited bandwidth of regional nodes and can carry out concurrent data transmission to the regional nodes with limited bandwidth at the current time, effectively alleviating the data transmission difficulties caused by link jitter, which provides technical supports for the braking control of the experimental object. The other two systems only adopt the simple broadcast confirmation method to screen regional nodes. Once a regional node has difficulties in data transmission due to limited bandwidth; it needs to be confirmed again in the next round of data transmission, which easily leads to network congestion. Therefore, the performance of data upload bandwidth of the system in this paper is significantly better than that of the other two systems.

The data packet loss frequency of the three systems in Rician channel and Gaussian channel is tested, and the test results are shown in Figure 9.

It can be seen from Figure 9 that the data packet loss frequency of the system in this paper is significantly lower than that of the other two systems when the signal-to-noise ratio increases. This is because the packet synchronization error control of the system in this paper improves the occurrence of control packet error; the other two systems use simple polling mechanism for error control in the process of data error packet control; that is, once a packet error occurs, the packet error will be discarded, resulting in the significant discard phenomenon of the other two systems in the event of signal jitter. The system in this paper has a significant advantage in controlling the packet loss frequency.

The speed and oil pressure control experiments are carried out to test the performance of the three systems. The control target value of oilfield minor repair machine' speed is set as $5.5 \mathrm{~m} / \mathrm{s}$, and the speed control results of three systems are compared as shown in Figure 10.

It can be seen from Figure 10 that the system can basically stabilize the speed of the experimental object around the set value of $5.5 \mathrm{~m} / \mathrm{s}$, and there is no large speed change in the control process. When there is a large disturbance, the system can make rapid adjustment, and the control results meet the technological requirements of workover operation. However, the other two systems cannot meet the requirement of $5.5 \mathrm{~m} / \mathrm{s}$ for the speed control target value of oilfield minor repair machine, so the speed control effect is poor, and the fluctuation is large.

The control target value of oil pressure of the oilfield minor repair machine is set as $1.8 \mathrm{MPa}$. Comparing the oil pressure control results of three systems, the results are as shown in Figure 11.

It can be seen from Figure 11 that the system in this paper is obviously superior to the other two systems in terms of oil pressure control. If the oil pressure of the oilfield minor repair machine is controlled at $1.8 \mathrm{MPa}$, the set oil pressure target can be achieved in a short time, which meets the control requirements of the oil pressure of the experimental object. However, the average oil pressure of the other two systems is controlled at $2.5 \mathrm{MPa}$ and $2.0 \mathrm{MPa}$, which does not reach the set target, and the control performance is poor. As can be seen from Figures 10 and 11, although the simulation results of the two models under different conditions are different, the trend is consistent, indicating that the method proposed in this paper has obvious advantages.

In order to further illustrate the advantages of this system, the experimental verification is carried out. Simulating the wet asphalt pavement, under the premise of the same parameters of other working conditions, the initial speed is $20 \mathrm{~m} / \mathrm{s}$. The oilfield minor repair machines controlled by three systems are used for braking, and the time and braking distance are measured when the vehicle speed is different. The measurement results are shown in Table 2.

It can be seen from Table 1 that, under the same initial conditions, the average control time of the system in this paper is $3.37 \mathrm{~s}$, which is $1.10 \mathrm{~s}$ and $0.81 \mathrm{~s}$ faster than the average braking time of the other two systems, respectively. The average braking distance of the system in this paper is $27.62 \mathrm{~m}$, which is $6.19 \mathrm{~m}$ and $2.12 \mathrm{~m}$ shorter than the average control distance of the other two systems, respectively. It shows that the control performance of the system is superior. The $K_{P}, K_{i}$ and $K_{D}$ of the system in this paper can be adjusted online under different braking conditions to achieve the purpose of optimizing the system performance index, greatly improving the robustness of the system to adapt to external interference. Compared with simple fuzzy control, the 


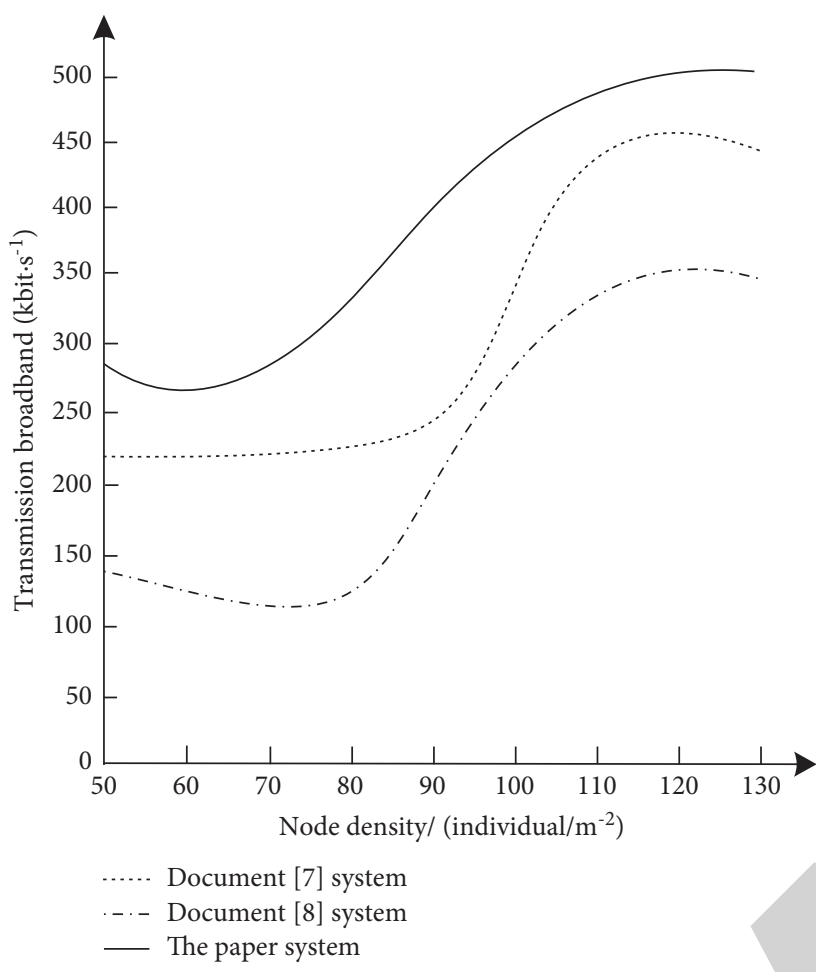

(a)

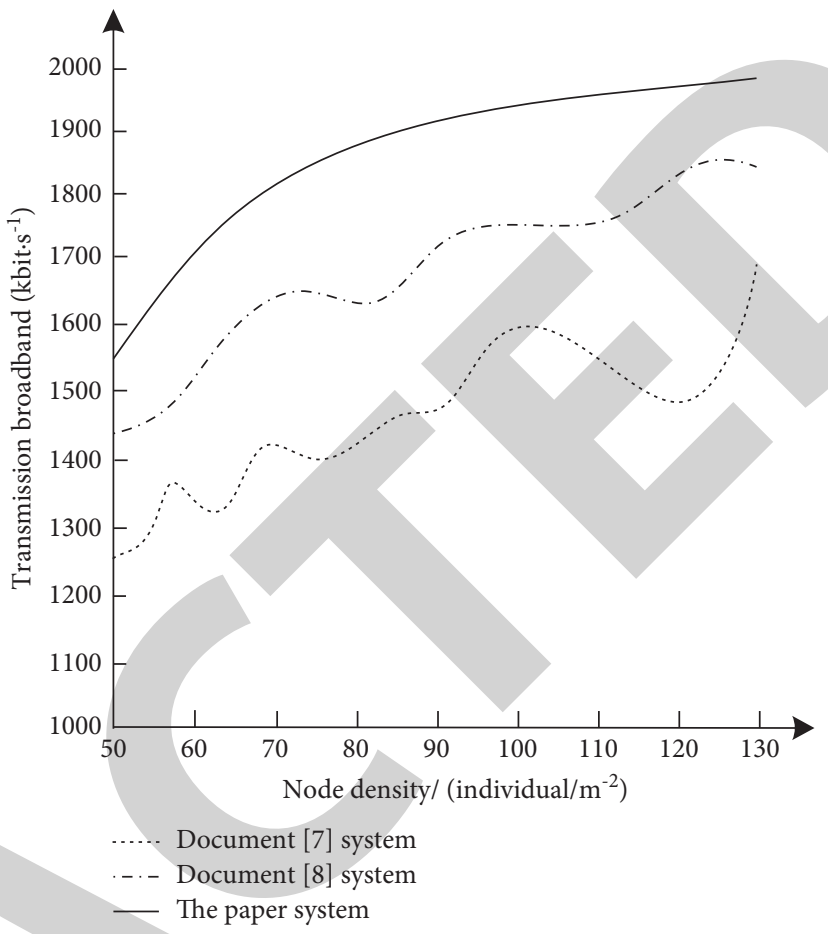

(b)

Figure 8: Data upload bandwidth. (a) Rician channel. (b) Gaussian channel.

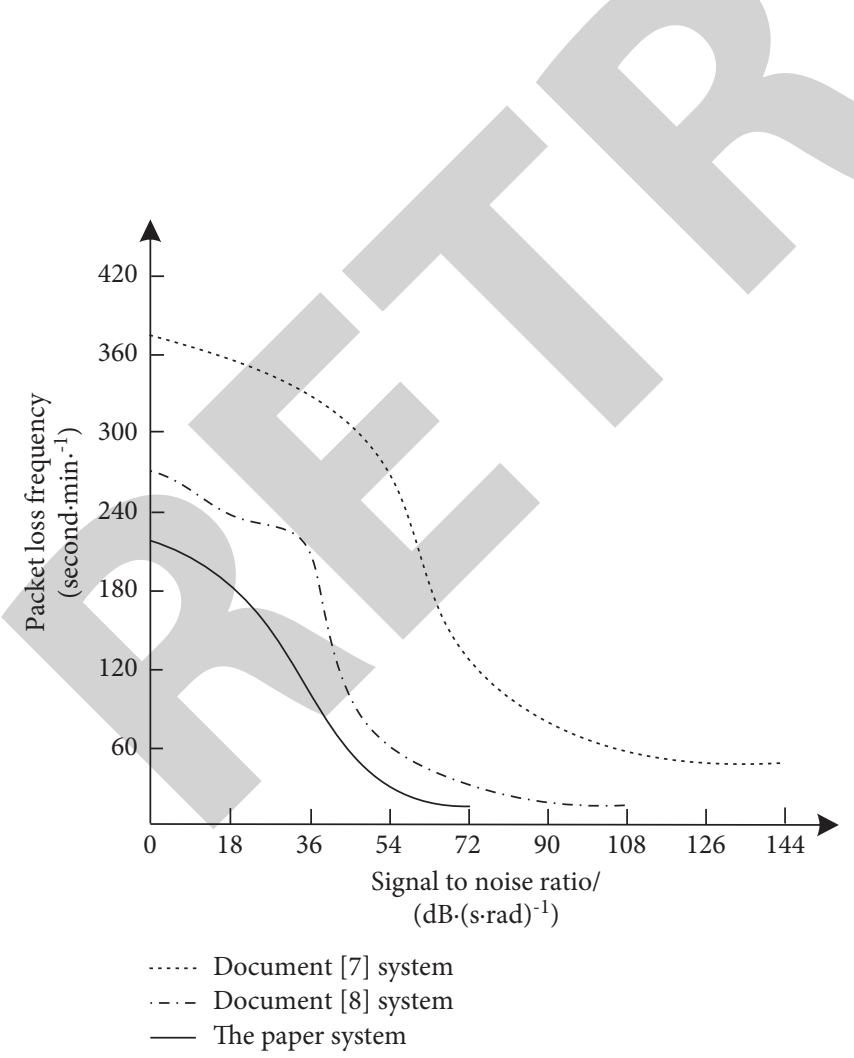

(a)

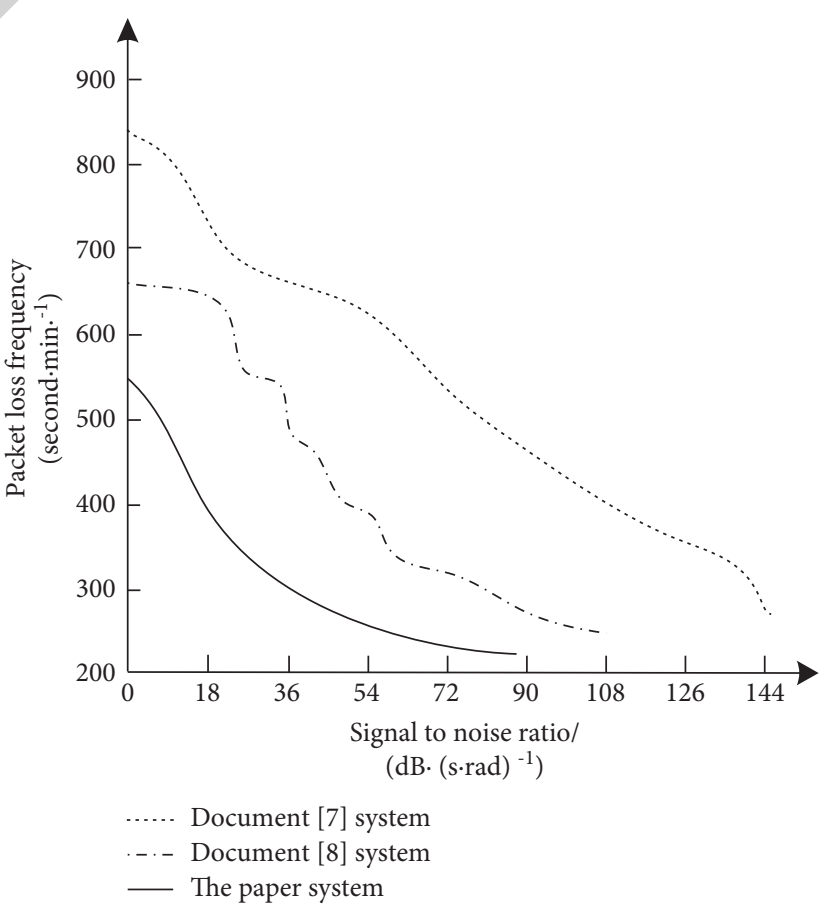

(b)

Figure 9: Packet loss frequency. (a) Rician channel. (b) Gaussian channel. 

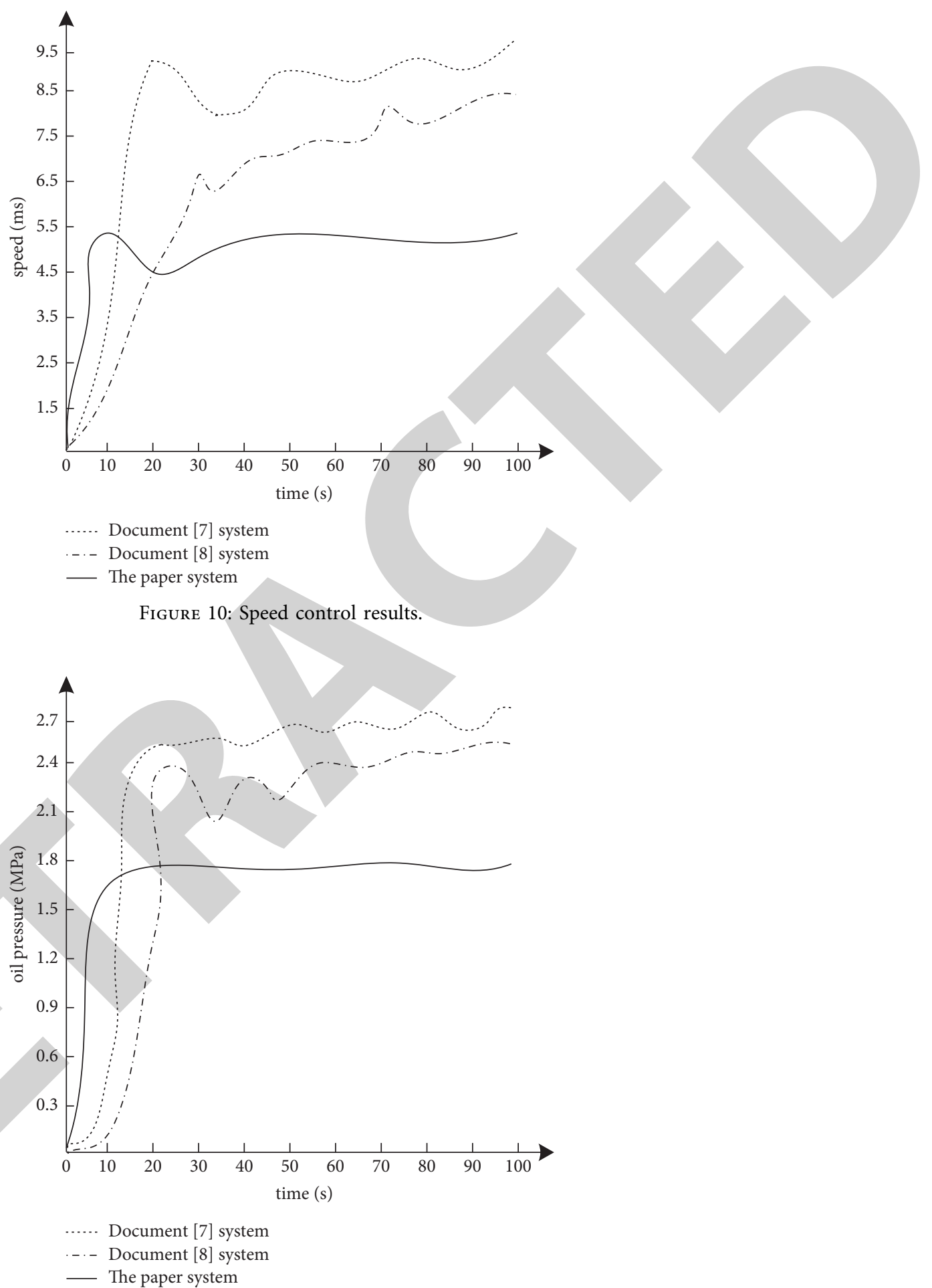

FIgURE 11: Results of oil pressure control.

dynamic deviation is reduced, and the stability of the equilibrium point is improved.

The results can be shown as follows in Figure 12. As shown in Figure 12, the blue bar represents our proposal, and on all oilfields, we can see that our proposal is better than others except $D$, in which all methods obtain the same results. It indicated that our proposal can perform well than the other three methods.
The results can be shown in Figure 12; the values of yellow bar represent the results of our proposal, and the others can be denoted as notations in Figure 4. And, on all datasets, we can see that our proposal is better than others except D4 (which represents the goal as 25), in which all methods obtain the same results. It indicated that our proposal can perform well than the other three methods. Comparative analysis of quality before and after 
TABLE 2: Comparison of experimental data.

\begin{tabular}{|c|c|c|c|c|c|c|}
\hline \multirow{2}{*}{ Speed $(\mathrm{m} / \mathrm{s})$} & \multicolumn{2}{|c|}{ Document [7] system } & \multicolumn{2}{|c|}{ Document [8] system } & \multicolumn{2}{|c|}{ The paper system } \\
\hline & Usage time (s) & Braking distance $(\mathrm{m})$ & Usage time (s) & Braking distance/m & Usage time (s) & Braking distance $(\mathrm{m})$ \\
\hline 20 & 3.22 & 32.56 & 2.88 & 28.44 & 2.72 & 26.97 \\
\hline 30 & 3.47 & 32.81 & 3.14 & 28.70 & 2.85 & 27.10 \\
\hline 40 & 3.72 & 33.06 & 3.40 & 28.96 & 2.98 & 27.23 \\
\hline 50 & 3.97 & 33.31 & 3.66 & 29.22 & 3.11 & 27.36 \\
\hline 60 & 4.22 & 33.56 & 3.92 & 29.48 & 3.24 & 27.49 \\
\hline 70 & 4.47 & 33.81 & 4.18 & 29.74 & 3.37 & 27.62 \\
\hline 80 & 4.72 & 34.06 & 4.44 & 30.00 & 3.50 & 27.75 \\
\hline 90 & 4.97 & 34.31 & 4.70 & 30.26 & 3.63 & 27.88 \\
\hline 100 & 5.22 & 34.56 & 4.96 & 30.52 & 3.76 & 28.01 \\
\hline 110 & 5.47 & 34.81 & 5.22 & 30.78 & 3.89 & 28.14 \\
\hline 120 & 5.72 & 35.06 & 5.48 & 31.04 & 4.02 & 28.27 \\
\hline
\end{tabular}

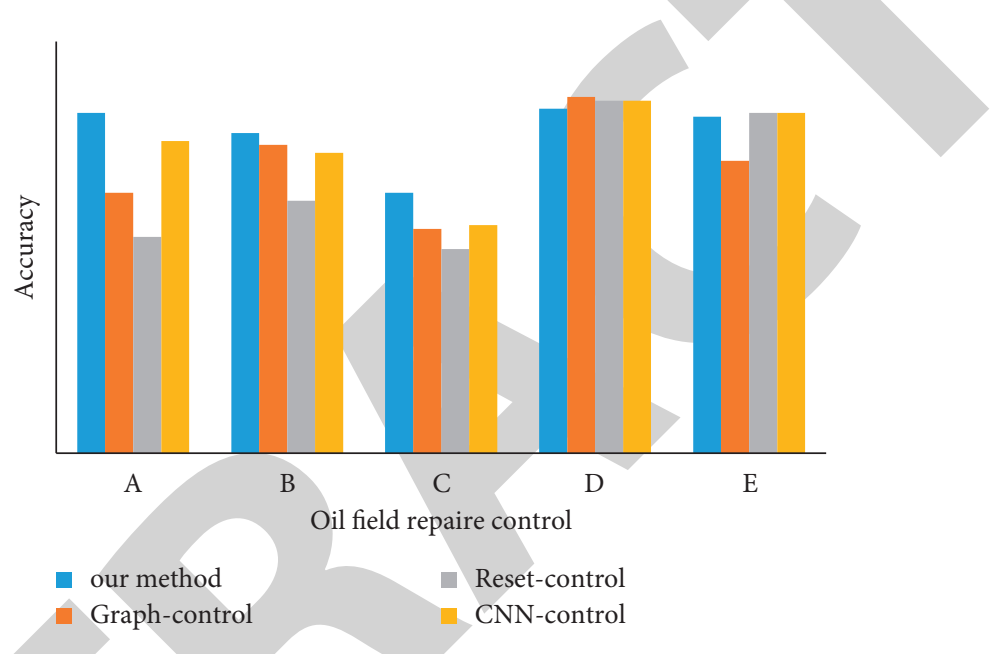

FIGURE 12: The results of all methods on oilfield control.

TABLE 3: MAE of all methods with various ratio of training and testing combination.

\begin{tabular}{lcccc}
\hline Proportion & Graph-control & Reset-control & CNN-control & Our proposal \\
\hline $6: 4$ & 0.859 & 0.896 & 0.955 & 0.763 \\
$7: 3$ & 0.854 & 0.832 & 0.841 & 0.741 \\
$8: 2$ & 0.852 & 0.811 & 0.845 & 0.726 \\
$9: 1$ & 0.847 & 0.766 & 0.823 & 0.709 \\
\hline
\end{tabular}

optimization is given in Figure 12. The minimum mass of the oilfield is the objective function, the thickness of the upper and lower plates is the design variable, and the stress intensity of the upper and lower plates is less than 340 as the constraint condition to optimize the size. After optimization, the safety factor is more than 1.4 except the braking condition, and the safety factor is still above 2 in the other conditions. Under the condition of not changing the structure of the control arm, the research and design of the available dimension optimization can quickly reduce the quality and guide the development and design of the oilfield repairing.

Under the above experimental environment parameters, the traditional methods and the wireless sensor based method in this paper are used for experiments, and the MAE results of all methods are obtained in the simulation software, as shown in Table 3:
The experimental results show that, with the increase of the proportion of training set and test set, the MAE values of the four algorithms are decreasing, and the accuracy is improving. In the case of each proportion, the MAE value of the proposed algorithm is lower than the three traditional methods, which shows that the recommendation accuracy and performance of the proposed method based on the Internet of things technology are higher.

\section{Conclusion}

In order to improve the braking control efficiency of oilfield minor repair machine, this paper designs the braking control system of oilfield minor repair machine based on wireless sensor network. Through the system design and functional performance test, we can get the following conclusions: 
(1) The braking control effect of the system of oilfield minor repair machine in this paper is good, and the system response is fast.

(2) The overall scheme of dividing the system into sensor network, front-end controller, remote-control center, and field control terminal is feasible and can meet the functional requirements of the project.

(3) Using CAN bus as the communication scheme of field sensor network can meet the reliability of field communication. 3G wireless communication module can carry out wireless communication normally and realize data transmission reliably. This system has significant advantages in data upload bandwidth performance and low control packet loss frequency.

The above conclusions show that the system can realize the collection and analysis of oilfield minor repair machine status information and remote brake control, achieve the expected design effect, and has certain engineering application value. However, in order to improve the brake control system of the oilfield minor repair machine based on the wireless sensor network and better meet the market demand, future research still needs to carry out the following work. A WiFi module is added to the front-end controller, and the WiFi module communicates with the local area network to realize wireless data transmission for on-site monitoring. According to on-site conditions, the front-end controller can be powered by new energy sources such as wind energy and solar energy in the work area permitted by the environment. Although our method has achieved better prediction accuracy than other popular methods, it still cannot achieve considerable accuracy when faced with a complex basketball game environment, and the training time of the model is long, which cannot meet the real goal time. In the future, we will further optimize the model to increase the training speed while ensuring accuracy.

\section{Data Availability}

The data used to support the findings of this study are available from the corresponding author upon request.

\section{Conflicts of Interest}

The authors declare that there are no conflicts of interest.

\section{Acknowledgments}

This paper was jointly sponsored by the National Natural Science Foundation of China (general project) (51174224) and the National Major Oil \& Gas Project (2016zx05066004002).

\section{References}

[1] L. Liu, G. Han, Z. Xu, J. Jiang, L. Shu, and M. Martinez-Garcia, "Boundary tracking of continuous objects based on binary tree structured SVM for industrial wireless sensor networks," IEEE Transactions on Mobile Computing, vol. 6, p. 1, 2020.
[2] Z. Zhang and Y. Zhang, "Application of wireless sensor network in dynamic linkage video surveillance system based on kalman filtering algorithm," The Journal of Supercomputing, vol. 75, no. 9, pp. 6055-6069, 2019.

[3] W. Wang and M. Zhang, "Self-adaptive gathering for energyefficient data stream in heterogeneous wireless sensor networks based on deep learning," IEEE Wireless Communications, vol. 27, no. 5, pp. 74-79, 2020.

[4] T. Wang, H. Xiong, H. Ding, and L. Zheng, "Tdoa-based joint synchronization and localization algorithm for asynchronous wireless sensor networks," IEEE Transactions on Communications, vol. 68, no. 5, pp. 3107-3124, 2020.

[5] S. M. Chowdhury and A. Hossain, "Impact of error control code on characteristic distance in wireless underground sensor networks," IET Communications, vol. 12, no. 13, pp. 1540-1549, 2018.

[6] B. J. Fan, Y. R. Li, and Y. Z. Liu, "The study on the controller of aviation switched reluctance starter/generator integrated system," Computer Simulation, vol. 36, no. 1, pp. 102-107, 2019.

[7] N. N. Tang, X. F. Zheng, J. Z. Wu, H. Chen, and H. X. Li, "Design of off-line led control system based on 4g-lte," Chinese Journal of Liquid Crystals and Displays, vol. 33, no. 1, pp. 55-60, 2018.

[8] B. H. Xiao, B. Q. Pei, and N. Wu, "Design of manned centrifuge control system based on motion controller," Space Medicine \& Medical Engineering, vol. 31, no. 1, pp. 32-36, 2018.

[9] S. Yang, Z. Gong, K. Ye, Y. Wei, Z. Huang, and Z. Huang, "EdgeRNN: a compact speech recognition network with spatio-temporal features for edge computing," IEEE Access, vol. 8, pp. 81468-81478, 2020.

[10] J. Zhang and D. Tao, "Empowering things with intelligence: a survey of the progress, challenges, and opportunities in artificial intelligence of things," IEEE Internet of Things Journal, vol. 8, no. 10, pp. 7789-7817, 2021.

[11] M. S. Hossain and G. Muhammad, "An audio-visual emotion recognition system using deep learning fusion for a cognitive wireless framework," IEEE Wireless Communications, vol. 26, no. 3, pp. 62-68, 2019.

[12] K. Khoshraftar and B. Heidari, "A hybrid method based on clustering to improve the reliability of the wireless sensor networks," Wireless Personal Communications, vol. 113, no. 2, pp. 1029-1049, 2020.

[13] Q. Liu, "Coverage reliability evaluation of wireless sensor network considering common cause failures based on $\mathrm{d}-\mathrm{s}$ evidence theory," IEEE Transactions on Reliability, vol. 70, no. 1, pp. 331-345, 2020.

[14] Y. Cui, L. Zhang, Y. Hou, and G. Tian, "Design of intelligent home pension service platform based on machine learning and wireless sensor network," Journal of Intelligent and Fuzzy Systems, vol. 40, no. 2, pp. 2529-2540, 2021.

[15] Z. Ying, J. Wang, and G. Hao, "An autonomous connectivity restoration algorithm based on finite state machine for wireless sensor-actor networks," Sensors, vol. 18, no. 2, p. 153, 2018.

[16] Y. Zhou, R. Zhao, Q. Luo, and C. Wen, "Sensor deployment scheme based on social spider optimization algorithm for wireless sensor networks," Neural Processing Letters, vol. 48, no. 1, pp. 71-94, 2018.

[17] X. Yu, H. Lu, X. Yang, Y. Chen, and W. Shi, “An adaptive method based on contextual anomaly detection in internet of things through wireless sensor networks," International 
Journal of Distributed Sensor Networks, vol. 16, no. 5, p. 155, 2020.

[18] R. Sharma, V. Vashisht, and U. Singh, "Woatca a secure and energy aware scheme based on whale optimisation in clustered wireless sensor networks," IET Communications, vol. 14, no. 8, pp. 1199-1208, 2020.

[19] J. Wang, R. Zhu, S. Liu, and Z. Cai, "Node location privacy protection based on differentially private grids in industrial wireless sensor networks," Sensors, vol. 18, no. 2, p. 410, 2018.

[20] Y. Yang, S. Wang, W. Xu, and K. Wei, "Reliability evaluation of wireless multimedia sensor networks based on instantaneous availability," International Journal of Distributed Sensor Networks, vol. 14, no. 11, p. 155, 2018.

[21] R. Tian, L. Wang, J. Zhang, H. Yang, and L. Zhang, "Wireless energy-efficient system based on the directed diffusion routing method for the high density seismic array survey," IOP Conference Series: Earth and Environmental Science, vol. 660, no. 1, Article ID 012140, 2021.

[22] S. Sivakumar and P. Vivekanandan, "Efficient fault-tolerant routing in iot wireless sensor networks based on path graph flow modeling with marchenko-pastur distribution (eftpmd)," Wireless Networks, vol. 26, no. 6, pp. 4543-4555, 2020.

[23] Y. Qi, C. Ma, H. Yu, and X. Bian, "A key pre-distribution scheme based on $\mu$-pbibd for enhancing resilience in wireless sensor networks," Sensors, vol. 18, no. 5, p. 1539, 2018.

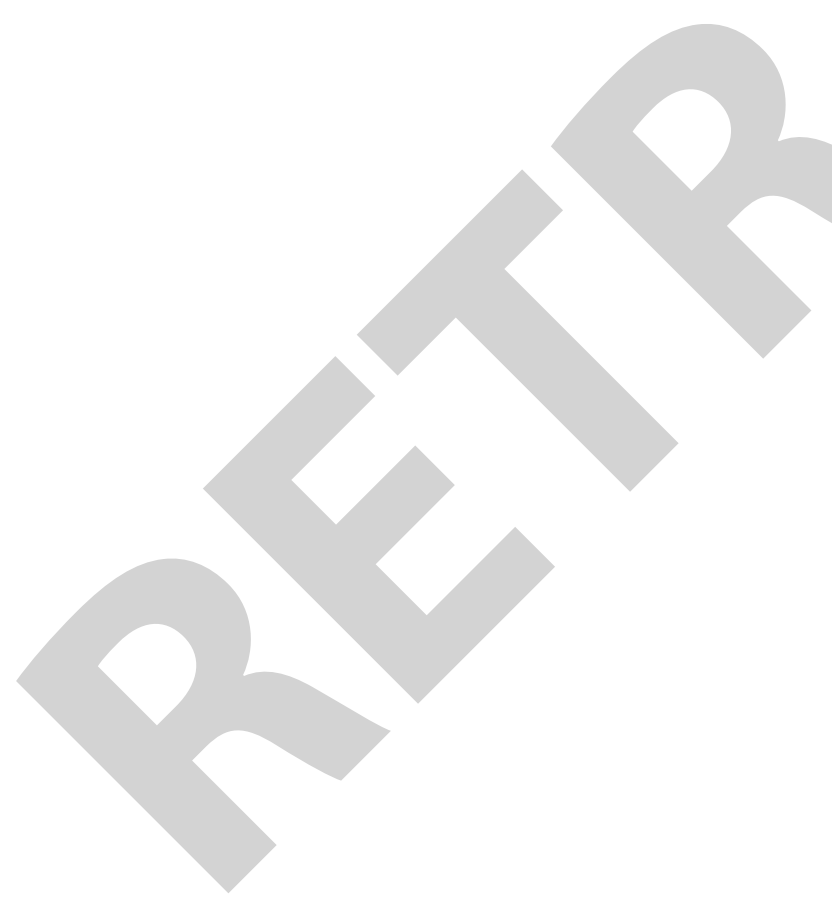

